\title{
Decreased circulatory microRNA-4478 as a specific biomarker for diagnosing non-ST-segment elevation myocardial infarction (NSTEMI) and its association with soluble leptin receptor
}

\author{
Gholikhani-Darbroud R ${ }^{1}$, Khaki-Khatibi $\mathrm{F}^{2}$, Mansouri $\mathrm{F}^{3}$, Hajahmadipoorrafsanjani $\mathrm{M}^{4}$, \\ Ghojazadeh $\mathrm{M}^{5}$
}

Department of Clinical Biochemistry, Faculty of Medicine, Tabriz University of Medical Sciences, Tabriz, Iran. fatemehkhakikhatibi@yahoo.com

\begin{abstract}
OBJECTIVE: This study aimed to assess the usefulness of circulatory microRNA-4478 (miR-4478) and soluble leptin receptor (SLEPR) in prognosis and diagnosis of non-ST-segment elevation myocardial infarction (NSTEMI) and to introduce miR-4478 as a new biomarker for NSTEMI disease. This study aimed also to examine correlation between miR-4478 and soluble leptin receptor and effects of miR-4478 on leptin receptor concentration. BACKGROUND: MicroRNAs could be used as predictive biomarkers for diseases.

METHODS: We collected sera of 80 angiographically confirmed NSTEMI patients and 80 healthy individuals and performed RNA extraction, cDNA synthesis, measurement of microRNAs, sLEPR and other chemistries. Statistical analyses were done using excel, XLSAT, and SPSS. Quality control analysis was done triplicated for 7 serial dilution of stock cDNA solution.

RESULTS: The patients with NSTEMI had higher serum levels of miR-4478, sLEPR, cTnI, CKMB, Urea, creatinine, glucose, cholesterol, TG, and ALP but lower levels of ALT compared with the normal healthy individuals. We detected decrease in expression of miR-4478 $\left(2^{\wedge}-\Delta \Delta C q=0.161 \pm 0.211\right)$ along with increase in sLEPR levels $(F=3.645, p<0.001)$ in the NSTEMI group compared with normal individuals. Pearson's correlation tests indicated positive correlation of miR-4478 and SLEPR $\left(p<0.001, R^{2}=0.698\right)$. There was sensitivity and specificity of $87.5 \%$ and $98.8 \%$ for $\mathrm{miR}-4478$ and $92.5 \%$ and $87.5 \%$ for sLEPR.

CONCLUSION: Circulatory miR-4478 and SLEPR may be used as predictors of NSTEMI. miR-4478 may also be used as a new biomarker for NSTEMI disease (Tab. 3, Fig. 6, Ref. 30). Text in PDF www.elis.sk.

KEY WORDS: microRNA-4478, Leptin receptor, non-ST-segment elevation myocardial infarction, biomarker.
\end{abstract}

\section{Introduction}

Cardiovascular disease (CVD), known as disorder of heart or blood vessels, has various types, depending on its mechanism of action and impact location. CVDs are classified to various cardiac implications and include: coronary artery diseases (CAD), stroke,

${ }^{1}$ Department of Clinical Biochemistry, Faculty of Medicine, Tabriz University of Medical Sciences, Tabriz, ${ }^{2}$ Drug Applied Research Center and Department of Clinical Biochemistry, Faculty of Medicine, Tabriz University of Medical Sciences, Tabriz, Iran, ${ }^{3}$ Cellular and Molecular Research Center and Department of Genetics and Immunology, Faculty of Medicine, Urmia University of Medical Sciences, Urmia, Iran, ${ }^{4}$ Cardiology Department, Seyedoshohada Cardiovascular Medical Hospital, Urmia University of Medical Sciences, Urmia, Iran, and ${ }^{5}$ Iranian Center for Evidence-Based Medicine, Tabriz University of Medical Sciences, Tabriz, Iran

Address for correspondence: F. Khaki-Khatibi, Drug Applied Research Center and Department of Clinical Biochemistry, Faculty of Medicine, Tabriz University of Medical Sciences, Tabriz, Iran.

Phone: +984133364666, Fax: +984133364666

Acknowledgement: This work was supported by the research Grant IR. TBZMED.REC 1395.43 of Tabriz University of Medical Sciences for final thesis of Ph.D students. heart failure, hypertensive heart disease, rheumatic heart disease, cardiomyopathy, heart arrhythmia, congenital heart disease, valvular heart disease, carditis, aortic aneurysms, peripheral artery disease, thromboembolic disease, and venous thrombosis (1).

CVDs are the leading cause of global death. In 2008, $30 \%$ of global death was attributed to cardiovascular diseases (2). A 2013 study in Iran showed a high incidence and mortality of CVD (331/100000 in men and 203/100000 in women) (3). CADs are responsible for about $12.2 \%$ of worldwide deaths in high- and middle-income countries (4).

Myocardial infarction (MI; commonly known as heart attack) and angina are presentations of CAD (also known as coronary heart disease (CHD) and ischemic heart disease (IHD)), but $\mathrm{MI}$ is more common. Risk factors for CADs include high blood pressure, lack of exercise, diabetes, smoking, obesity, high LDL/ HDL among others $(5,6)$. A number of tests are used to diagnosis CADs, including electrocardiography (ECG), coronary angiography, and some blood tests like cardiac troponin I and T, and less often creatine kinase MB (CK-MB) (7). Increase or not-increase in ST segment on ECG (along with the presence of other signs and symptoms of MI) is the main criterion to classify MI to ST 
elevation MI (STEMI) and non-ST elevation MI (NSTEMI), respectively (8). Although a recent classification is presented, STEMI and NSTEMI terms are more accepted and used by health organizations and cardiologists (9).

In contrast to STEMI where the artery is almost totally occluded by clots rich in fibrin and cholesterol that obligates immediate management, the infarct-related artery is partially occluded in NSTEMI. Quick and straightaway diagnosis is important and lifesaving by initiating appropriate therapy to control development of MI and reduce the mortality rate (10).

Leptin receptor (LEPR, OB-R) is a member of cytokine receptor family present in hypothalamic neurons that with the physiologic function of controlling hunger and energy use by binding a fat cell-specific hormone leptin (11). The soluble form of it (sLEPR, sOB-R) that results from cleavage of the extracellular domain of the transmembraneous leptin receptor, has 839 amino acids and may be detected in the circulatory system (12). Circulatory LEPR has two distinctly separate $\mathrm{N}$-glycosylated isoforms and may be present in dimeric or oligomerized states (13). By binding to it in ratio of 1: 1, sLEPR modulates leptin action (14).

Increase in the circulatory leptin receptor and its ligand have been demonstrated in chronic heart failure (15).

Recently along with advances in molecular medicine, nucleotide-based biomarkers for the diagnosis or prognosis of diseases have attracted significant attention. MicroRNAs (miRNAs) are endogenous, single stranded, non-coding RNA particles of 19-25 nucleotides long, encoded by a specific transcription and splicing mechanisms present in both nucleus and cytosol (16). MiRNAs play their critical roles in silencing of specific mRNAs by binding to the 3'-untranslated region (3' UTR) of target mRNA and inhibiting their translation or inducing their degradation (17). MiRNAs are involved in several biochemical processes in the cell, including proliferation, differentiation, development, migration, and apoptosis (18). MiRNAs are excreted to body fluids bounded to specific proteins that give them enough stability to be detected. Various conditions, especially diseases cause excretion of miRNAs from tissues to the body fluids, so variations in their body-fluid levels may be a representation of their variation in the tissue. So miRNAs (specially tissue specific ones) may be used a biomarkers for various diseases, including cardiovascular diseases, and their increase or decrease in tissue or body fluids (including serum) may be used as useful prognostic factors for disease. Some muscle-specific microRNA (called myomiRs or miRs) that would change in tissue-specific disorders, including heart diseases are: miR-1, miR-133a, miR-206, miR-208a, miR-208b, miR-499, and miR-486 (19).

Micro RNA 4478 (miR-4478, Chromosomal location: 9q33.2) has the highest target score $(=90)$ for LEPR (20), so it could be easily deduced that variation in miR-4478 (reflected by changes in its serum level) may affect serum LEPR concentration.

The objectives of this study were evaluating circulatory miR4478 and sLEPR differentiation between NSTEMI patients and healthy control subjects, calculating the effects of changes in miR4478 on LEPR level, and investigating the role of miR-4478 as a marker for predicting NSTEMI.

\section{Materials and methods}

\section{Ethics statement}

The study was approved by the Ethic Committee of Medicine faculty of Tabriz University of Medical Sciences. Blood collection procedures from patients and control groups complied completely with relevant rules of Seyedoshohada Cardiovascular Medical Hospital of Urmia University of Medical Sciences. Before participating in the study, a written informed consent was obtained from each patient and healthy individual that was enrolled in the current study.

\section{Study population}

80 hospitalized patients (40 males (50\%) and 40 females (50 $\%$ ) with age range of 35-80 suffering from NSTEMI with their disease diagnosed by the cardiologists of Seyedoshohada Cardiovascular Medical Hospital were included in the patient group during May 2016 and October 2016. 80 individuals with equal age and sex range referred to the hospital that had normal angiographic and electrocardiographic results were enrolled as control group during the same time period. Patients with a history of malignant disease, liver failure, renal failure, previous history of cardiomyopathy, bleeding disorders and irradiation therapy were excluded from the study. The study was conducted in accordance with WMA Declaration of Helsinki - Ethical Principles for Medical Research and guidelines of the ethics committee of Tabriz University of Medical sciences and Seyedoshohada Cardiovascular Medical Hospital of Urmia, Iran.

\section{Sample collection and storage}

Blood samples were collected in mediplus clot-activator containing tubes (Sunphoria Co. Ltd., Taiwan) and left for 10 minutes to complete coagulation and then sera were separated by centrifugation at $10000 \mathrm{rpm}$ for 7 minutes, and transferred to RNase/ DNase-free tubes and stored frozen at $-80{ }^{\circ} \mathrm{C}$ (snijders scientific, The Netherlands) until analysis.

\section{RNA isolation and preparation}

Large and small (including micro-) RNAs in serum was isolated in separate tubes using Hybrid-R miRNA Purification Kit (Geneall, Seoul, South Korea) according to instructions of the manufacturer. The procedure includes inactivation of nucleases by RiboEx Buffer (containing phenol and guanidium salt), followed by adding chloroform to push RNAs to aqueous phase (DNA particles remain in interphase and proteins remain in organic phase). Ethanol was added to aqueous phase and large and small RNAs separated, respectively, by type W and type B columns. At last, RNAs were excluded from mentioned columns by RNase/ DNase-free water and kept frozen at $-80{ }^{\circ} \mathrm{C}$.

\section{Quantification of miR-4478}

Total RNA had been polyadenylated and reverse transcribed to make cDNA using the NG dART RT kit (EURx Ltd., Gdansk, Poland) following the manufacturer instructions. As brief, $0.5 \mu \mathrm{g}$ of the DNase-treated total RNA as template was mixed with $1 \mu \mathrm{l}$ NG dART RT mix, $1 \mu$ Oligo (dT) ${ }_{20}$ primed primer, $4 \mu$ of $5 \times$ NG 
cDNA buffer, and reached to final volume of $20 \mu \mathrm{l}$ by RNase-free water. The mixture was transferred to preheated thermal cycler and incubated at $50{ }^{\circ} \mathrm{C}$ for 50 minutes to complete the reaction and at $85{ }^{\circ} \mathrm{C}$ for 5 minutes to stop it. The obtained cDNA (2.5 $\mu 1$ of a 1/100 dilution) was then amplified with real-time PCR using MIC qPCR Cycler (biomolecular systems, Australia). Human mature miR-4478 has 17 nucleotides with this sequence: 3'-GAGGCUGAGCUGAGGAG-5'. Primer sets for miR-4478 (hsa-miR-4478 LNA ${ }^{\text {TM }}$ PCR primer set, Product no. 2108950), housekeeping internal control, and master mix were shipped from Exiqon (Vedbaek, Denmark). Considering Exiqon recommendations, we used microRNA-423-5p (hsa-miR-423-5pLNA ${ }^{\text {TM }}$ PCR primer set, Product no. 205624) as the endogenous housekeeping internal control and ExiLENT SYBR ${ }^{\circledR}$ Green master mix (Product no. 203403) as the master mix.

PCR reactions were conducted at $95{ }^{\circ} \mathrm{C}$ for 15 minutes, followed by 40 cycles of $60.5^{\circ} \mathrm{C}$ for 20 seconds, and $72{ }^{\circ} \mathrm{C}$ for 20 seconds. A melting curve analysis was settled to confirm that there is only one specific peak for miR-4478 and to validate the specificity of PCR product according to the MIQE guidelines (21). The quantification cycle $(\mathrm{Cq})$ values were calculated for each of these: Gene being Tested Experimental (TE.Cq), Gene being Tested Control (TC.Cq), Housekeeping gene Experimental (HE. $\mathrm{Cq}$ ), and Housekeeping gene Control (HC.Cq). "TE.Cq - HE.Cq" was measured as " $\Delta$ Cq.E" and "TC.Cq - HC.Cq" as " $\Delta$ Cq.C". " $2^{\wedge}-\Delta$ Cq.E" and " $2^{\wedge}-\Delta$ Cq. $C$ " values were also calculated to better comparison. No enzyme control and no template control (NTC) were included among with case and control samples as the negative controls to discover any potential DNA and/or water/ master mix contamination, respectively. All qRT-PCR reactions (samples, controls, and negative controls) were performed in triplicate and means of them were used as $\mathrm{Cq}$ values for each.

\section{Detection of soluble leptin receptor}

We used an Enzyme Linked Immunosorbent Assay kit (ELISA) (Zellbio, Germany; Cat. No. ZB-10304-H9648) and followed kit instructions to measure the sLEPR level in NSTEMI patients and healthy control subjects.

\section{Measurement of other circulating substances}

Other circulating chemistries were measured by an autoanalyzer system (Autolab, BT 3500, Auto analyzer Medical System, Rome, Italy) and chemiluminescent analyzer (Architect i1000 SR, Abbott, USA) using commercial kits (Pars Azmoon, Tehran, Iran) in the clinical laboratory of Seyedoshohada Cardiovascular Medical Hospital.

\section{Statistical analysis}

Analysis of relative gene expression data was done using the $2^{-\Delta \Delta \mathrm{Cq}}$ method $(21-24)$. LinReg PCR method $(25,26)$ in program software was chosen to determine the reaction effectiveness for each primer pair. Microsoft Excel version 2013 (Microsoft Corp., Redmond, WA, USA), XLSTAT Version 2016.02.28451 (Addinsoft, Paris, France) and SPSS version 20 (SPSS Inc., Chicago, IL, USA) softwares were applied to statistical analysis and generation
Tab. 1. Clinical characteristics of patient and control groups.

\begin{tabular}{lccc}
\hline Characteristics & $\begin{array}{c}\text { NSTEMI } \\
\text { Patients } \\
(\mathrm{n}=80)\end{array}$ & $\begin{array}{c}\text { Healthy } \\
\text { Controls } \\
(\mathrm{n}=80)\end{array}$ & $\begin{array}{c}\text { Two-tailed } \\
\text { p value } \\
(\mathrm{t}-T e s t)\end{array}$ \\
\hline Age $($ years) & $62.40 \pm 9.86$ & $59.61 \pm 10.83$ & 0.091 \\
Male/Female & $40 / 40$ & $40 / 40$ & 1 \\
Body mass index $\left(\mathrm{kg} / \mathrm{m}^{2}\right)$ & $25.12 \pm 4.57$ & $24.89 \pm 4.24$ & 0.460 \\
cTnI $(\mathrm{ng} / \mathrm{l})$ & $18.90 \pm 48.68$ & $0.13 \pm 0.11$ & $\mathbf{0 . 0 0 1}$ \\
CKMB $(\mathrm{IU} / \mathrm{l})$ & $32.87 \pm 22.53$ & $17.92 \pm 6.21$ & $<\mathbf{0 . 0 0 1}$ \\
Urea $(\mathrm{mg} / \mathrm{dl})$ & $37.47 \pm 14.70$ & $28.56 \pm 7.12$ & $<\mathbf{0 . 0 0 1}$ \\
Creatinine $(\mathrm{mg} / \mathrm{dl})$ & $1.15 \pm 0.29$ & $1.06 \pm 0.15$ & $\mathbf{0 . 0 0 9}$ \\
Glucose $(\mathrm{mg} / \mathrm{dl})$ & $144.26 \pm 65.96$ & $103.85 \pm 17.50$ & $<\mathbf{0 . 0 0 1}$ \\
Na ${ }^{+}(\mathrm{mEq} / \mathrm{l})$ & $140.97 \pm 1.60$ & $140.88 \pm 1.58$ & 0.729 \\
$\mathrm{~K}^{+}(\mathrm{mEq} / \mathrm{l})$ & $4.17 \pm 0.34$ & $4.24 \pm 0.32$ & 0.194 \\
Cholesterol $(\mathrm{mg} / \mathrm{dl})$ & $182.92 \pm 37.44$ & $165.35 \pm 22.59$ & $<\mathbf{0 . 0 0 1}$ \\
TG $(\mathrm{mg} / \mathrm{dl})$ & $183.70 \pm 50.43$ & $160.22 \pm 36.02$ & $\mathbf{0 . 0 0 1}$ \\
HDL $(\mathrm{mg} / \mathrm{dl})$ & $39.17 \pm 8.39$ & $39.51 \pm 9.36$ & 0.811 \\
AST & $24.81 \pm 27.39$ & $25.70 \pm 21.37$ & 0.820 \\
ALT & $22.86 \pm 14.68$ & $30.86 \pm 25.94$ & $\mathbf{0 . 0 1 8}$ \\
ALP & $214.36 \pm 51.52$ & $200.83 \pm 51.68$ & 0.099 \\
LDL & $94.26 \pm 40.13$ & $98.53 \pm 38.12$ & 0.491 \\
TSH & $3.69 \pm 11.64$ & $2.60 \pm 9.09$ & 0.511 \\
FT4 & $1.80 \pm 1.78$ & $2.02 \pm 1.90$ & 0.443 \\
\hline
\end{tabular}

$\mathrm{cTnI}=$ cardiac troponin $\mathrm{I}, \mathrm{CKMB}=$ Creatine Kinase-MB isozyme, $\mathrm{Na}^{+}=$sodium, $\mathrm{K}^{+}=$potassium, $\mathrm{TG}=$ Triglyceride, $\mathrm{HDL}=$ high density lipoprotein, $\mathrm{AST}=$ aspartate transaminase, $\mathrm{ALT}=$ alanine transaminase, $\mathrm{ALP}=$ alkaline phosphatase, $\mathrm{LDL}=$ low-density lipoprotein, TSH $=$ Thyroid-stimulating hormone, FT4 = free thyroxine

of presented graphs. Sample size was big enough to accept normal distribution of study groups, but we performed a KolmogorovSmirnov normality test (K-S test) for confirmation. Two-tailed $\mathrm{p}<0.005$ was considered statistically as significant. Correlation between miR-4478 expression and SLEPR was estimated using Pearson correlation coefficient. To determine the sensitivity and specificity of changes in miR-4478 expression as a discriminative predictor of NSTEMI disease, a Receiver Operating Characteristic (ROC) curve analysis was employed.

\section{Results}

\section{Subject characteristics}

Characteristics and some routine analyses of NSTEMI patients and healthy controls included in the current study are presented briefly in Table 1. Other tests that have been done in some of the included individuals were T3, T3up, HbA1c, Albumin, Amylase, CPK, Iron, TIBC, Uric acid, and VLDL, but their sample sizes were below 10 for patients and/or controls and then, analytical statistics were not done in them. No significant differences were observed in age, sex, BMI, and serum chemistries like sodium, potassium, HDL, AST, LDL, TSH, and FT4 levels. Nevertheless, patients with NSTEMI had higher serum concentrations of miR4478 (shown by DeltaCqs), sLEPR, cTnI, CKMB, Urea, creatinine, glucose, cholesterol, TG, and ALP but lower levels of ALT compared with the normal healthy individuals.

Gene expression pattern of miR-4478 in NSTEMI patients and normal healthy individuals

Analyses of serum miR-4478 expressions were done by qPCR in all studied cases. Quality control analysis was done three 


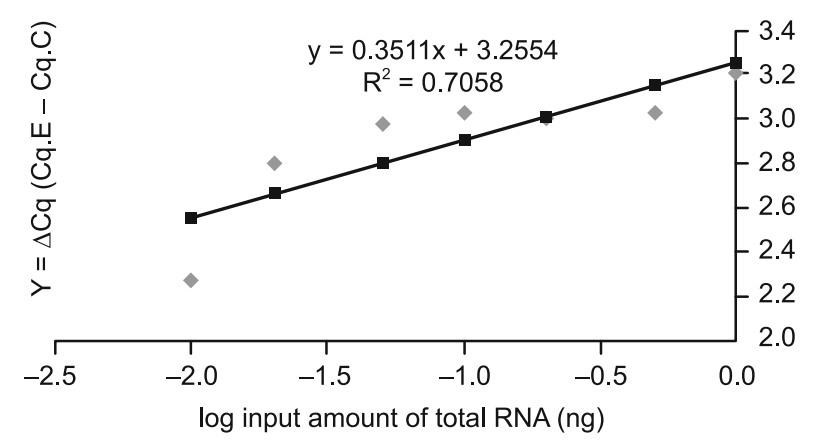

$$
\text { - Y - Predicted L Linear }(\mathrm{Y})
$$

Fig. 1. Logarithm of input amount of serial dilutions of total RNA against their corresponding $\Delta$ Cqs. $\mathbf{R}^{2}=$ Coefficients of Determination.

timese for each of 7 serial dilutions of stock cDNA solutions as follows: $1,0.5,0.2,0.1,0.05,0.02$, and 0.01 . Results of regression analysis on logarithm of these dilutions and their corresponding $\Delta$ Cqs plotted in Figure 1, show trustworthy of the qPCR analysis. Comparison of the mean values of $\Delta$ Cq.E and $\Delta$ Cq.C as well as $2^{\wedge}-\Delta$ Cq.E and $2^{\wedge}-\Delta$ Cq.C along with calculation of $2^{\wedge}-\Delta \Delta \mathrm{Cq}$ (Fold Change in Gene Expression) indicated statistically lower expression of miR-4478 in the NSTEMI group compared with healthy group (Figs 2 and 3).
Serum level of SLEPR in NSTEMI patients and normal healthy group

ELISA analyses results showed a significant increase in SLEPR levels of NSTEMI patients $(30.568 \pm 3.290 \mathrm{ng} / \mathrm{dl})$ compared with normal healthy individuals $(23.740 \pm 5.457 \mathrm{ng} / \mathrm{dl})(\mathrm{F}=3.645, \mathrm{p}$ $<0.001)$.

The predictive value of serum miR-4478 and SLEPR for NSTEMI

Receiver operating characteristic curves (ROC curves) were used to measure the sensitivity and specificity of the test as well as to predict the efficiency for NSTEMI disease, and the area under the ROC curve (AUC) was used to measure accuracy of the test. Correlation test (Pearson's) and linear regression test were also done.

Correlation test (Pearson's) showed correlation of -0.835 between $2^{\wedge}-\Delta \Delta \mathrm{Cq}$ (Fold Change in Gene Expression) and sLEPR level $(\mathrm{p}<0.001)$. Linear regression test showed $\mathrm{R}^{2}=0.698$ with model equation of LEPR $=32.658-13.00 \times 2^{\wedge}-\Delta \Delta \mathrm{Cq}$ (Fig. 4).

The ROC curve analyses for $2^{\wedge}-\Delta \mathrm{Cq}$ showed high sensitivity $(87.5 \%)$ and specificity $(98.8 \%)$ with $\mathrm{AUC}=0.936$ and accuracy $=0.931(95 \%$ confidence interval on the difference between the AUC and 0.5 (Two-tailed test) was 0.395-0.476, p-value (Twotailed) $<0.0001)$ (Fig. 5).

The ROC curve analyses for sLEPR also showed high sensitivity $(92.5 \%)$ and specificity $(87.5 \%)$. AUC was equal to 0.862 and accuracy was equal to 0.900 (95\% confidence interval was 0.295-0.430, p-value (Two-tailed) < 0.0001) (Fig. 5).
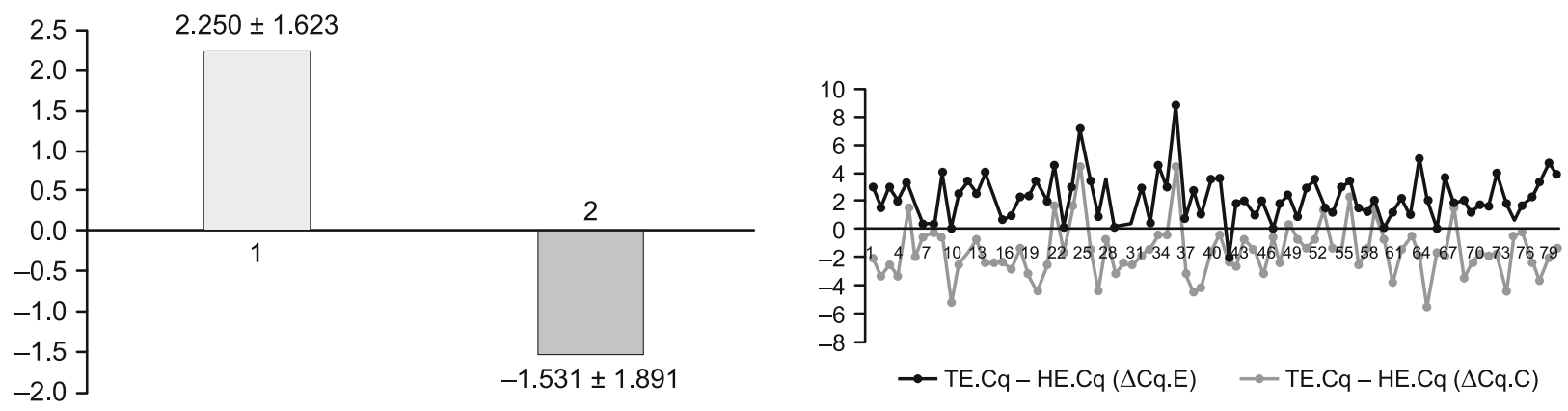

Fig. 2. Mean \pm SD for $\Delta$ Cq.E and $\Delta$ Cq.C $(<0.0001) . \Delta C q . E=$ Difference between mean Cqs of experimental and housekeeping gene for patients, $\Delta$ Cq.C $=$ Difference between mean Cqs of experimental and housekeeping gene for healthy controls, SD $=$ Standard Deviation.
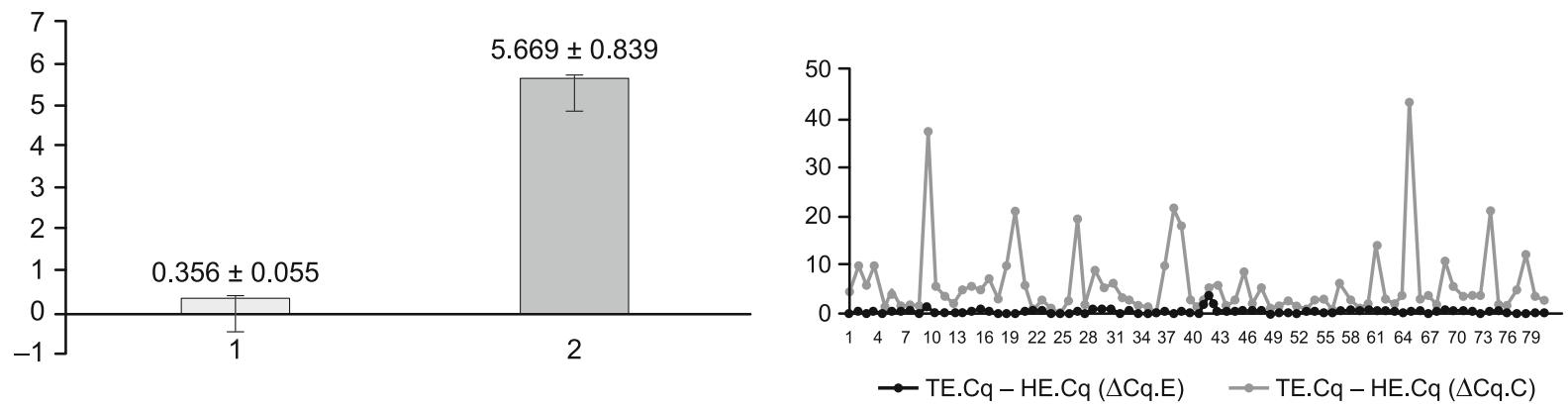

Fig. 3. Differences between Mean \pm SD levels by comparison of $2^{\wedge}-\Delta C q . E$ and $2^{\wedge}-\Delta C$ Cq.C $(p<0.0001) .2^{\wedge}-\Delta$ Cq.E $=2$ power by minus $\Delta$ Cq.E, $2^{\wedge}-\Delta$ Cq.C $=2$ power by minus $\Delta$ Cq.C,, $\mathbf{S D}=$ Standard Deviation. 


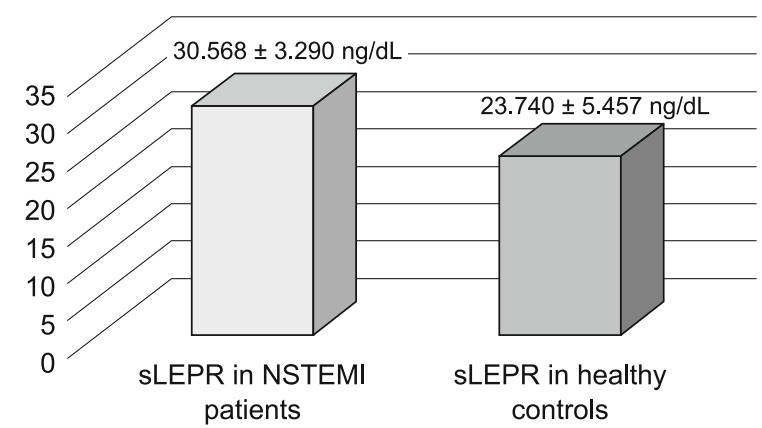

Fig. 4. Fig. 4. sLEPR levels of NSTEMI patients compared with normal healthy individuals $(\mathrm{F}=3.645, p<0.0001)$.

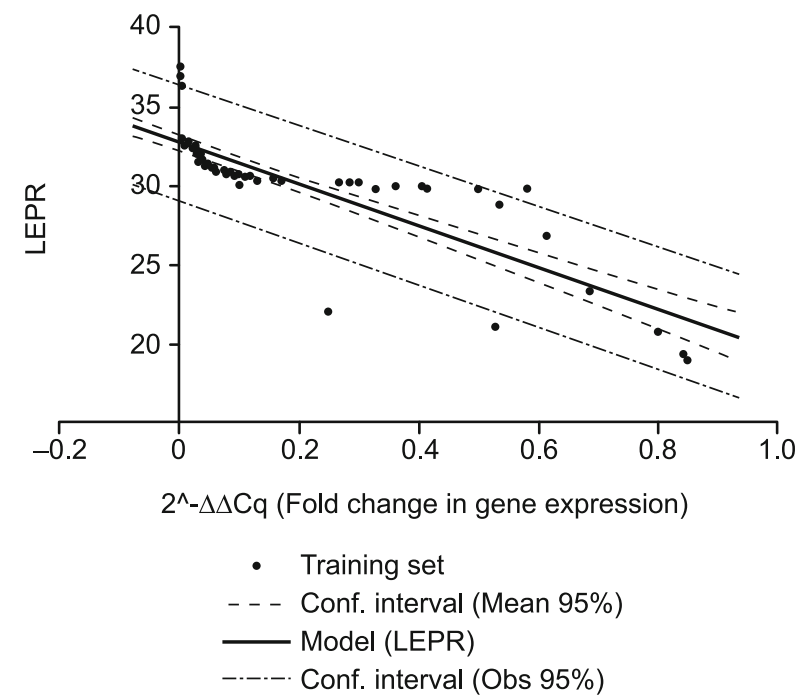

Fig. 5. Correlation and linear regression of $2^{\wedge}-\Delta \Delta C q$ against soluble LEPR. $2^{\wedge}-\Delta \Delta C q=2$ power by minus difference between $\Delta C \mathrm{Cq} . \mathrm{E}$ and $\triangle$ Cq.C, LEPR $=$ Leptin Receptor, Conf. interval $=$ Confidence Interval, Obs $=$ Observed, $\mathbf{R}^{2}=$ Coefficients of Determination .

Correlations between miR-4478 and sLEPR and other variables

Correlation and linear regression of $2^{\wedge}-\Delta \Delta C q$ as indicant of miR-4478 gene expression level against soluble LEPR is shown in Figure $6\left(\mathrm{p}<0.001, \mathrm{R}^{2}=0.698\right)$. Correlation and linear regression between $2^{\wedge}-\Delta \Delta C q$ and sLEPR with other chemistries that have significant variation between patient and control groups (CKMB, Urea, Creatinine, Glucose, $\mathrm{Na}^{+}, \mathrm{K}^{+}$, Cholesterol, TG, HDL, and AST) were statistically compared. As shown in Table 3, there is statistically significant reverse correlation between fold change in expression of miR-4478 and serum concentrations of CKMB, and glucose. Similarly, there is a significant positive correlation between sLEPR and cTnI, CKMB, urea, glucose and cholesterol, and reverse correlation with ALT.

\section{Discussion}

Coronary artery diseases (CADs) are the most common and serious class of cardiovascular diseases (CVDs). Among CADs,
Tab. 2. Statistical results of independent t-test for changes in expression of miR-4478 in NSTEMI patients in comparison with control healthy group.

\begin{tabular}{lccc}
\hline & No. & $\begin{array}{c}\text { Mean } \pm \text { Standard } \\
\text { Deviation (SD) }\end{array}$ & Description \\
\hline TE.Cq - HE.Cq $(\Delta$ Cq.E) & 80 & $2.250 \pm 1.623$ & $\mathrm{p}$ (Two-tailed) $<0.0001$ \\
TC.Cq - HC.Cq $(\Delta$ Cq.C) & 80 & $-1.531 \pm 1.891$ & \\
\hline $2^{\wedge}-\Delta$ Cq.E & 80 & $0.356 \pm 0.489$ & $\mathrm{p}$ (Two-tailed) $<0.0001$ \\
$2^{\wedge}-\Delta$ Cq.C & 80 & $5.669 \pm 7.505$ & \\
\hline $2^{\wedge}-\Delta \Delta$ Cq (fold change) & 80 & $0.161 \pm 0.211$ & $\begin{array}{c}\text { Fold change is below } \\
\text { basement }(1)\end{array}$ \\
\hline $\log 2^{\wedge}-\Delta \Delta$ Cq & 80 & $-1.138 \pm 0.569$ & $\begin{array}{c}\text { Log of fold change is be- } \\
\text { low log of basement }(0)\end{array}$ \\
\hline
\end{tabular}

$\Delta \mathrm{Cq} . \mathrm{E}=$ Difference between mean Cqs of experimental and housekeeping gene for patients, $\Delta \mathrm{Cq} \cdot \mathrm{C}=$ Difference between mean Cqs of experimental and housekeeping gene for healthy controls, $2^{\wedge}-\Delta \mathrm{Cq} . \mathrm{E}=2$ power by minus $\Delta \mathrm{Cq} . \mathrm{E}, 2^{\wedge}-\Delta \mathrm{Cq} \cdot \mathrm{C}=2$ power by minus $\Delta$ Cq.C, $2^{\wedge}-\Delta \Delta \mathrm{Cq}=2$ power by minus difference between $\Delta \mathrm{Cq}$.E and $\Delta \mathrm{Cq}$.C, $\log 2^{\wedge}-\Delta \Delta \mathrm{Cq}=$ logarithm of $2^{\wedge}-\Delta \Delta \mathrm{Cq}, \mathrm{SD}=$ Standard Deviation

Tab. 3. Correlation of sLEPR and expression fold change in miR-4478 with some variables that were statistically different between patient and control groups. Numbers in bold represent significant correlation.

\begin{tabular}{|c|c|c|c|}
\hline & $\mathrm{p}$ & Pearson's $r$ & $\mathrm{R}^{2}$ \\
\hline $\begin{array}{l}2^{\wedge}-\Delta \mathrm{Cq} \\
\mathrm{cTnI}\end{array}$ & 0.160 & -0.112 & 0.012 \\
\hline $\begin{array}{l}2^{\wedge}-\Delta \mathrm{Cq} \\
\text { CKMB }\end{array}$ & 0.004 & -0.224 & 0.050 \\
\hline $\begin{array}{l}2^{\wedge}-\Delta \mathrm{Cq} \\
\text { Urea }\end{array}$ & 0.088 & -0.135 & 0.018 \\
\hline $\begin{array}{l}2^{\wedge}-\Delta \mathrm{Cq} \\
\text { Creatinine } \\
\end{array}$ & 0.157 & -0.112 & 0.013 \\
\hline $\begin{array}{l}2^{\wedge}-\Delta \mathrm{Cq} \\
\text { Glucose } \\
\end{array}$ & 0.033 & -0.169 & 0.029 \\
\hline $\begin{array}{l}2^{\wedge}-\Delta \mathrm{Cq} \\
\text { Cholesterol }\end{array}$ & 0.283 & -0.085 & 0.007 \\
\hline $\begin{array}{l}2^{\wedge}-\Delta \mathrm{Cq} \\
\mathrm{TG} \\
\end{array}$ & 0.087 & -0.136 & 0.018 \\
\hline $\begin{array}{l}2^{\wedge}-\Delta \mathrm{Cq} \\
\text { ALT } \\
\end{array}$ & 0.063 & 0.147 & 0.022 \\
\hline $\begin{array}{l}\text { sLEPR } \\
\text { cTnI }\end{array}$ & 0.009 & 0.206 & 0.04 \\
\hline $\begin{array}{l}\text { sLEPR } \\
\text { CKMB } \\
\end{array}$ & 0.041 & 0.162 & 0.026 \\
\hline $\begin{array}{l}\text { sLEPR } \\
\text { Urea } \\
\end{array}$ & 0.005 & 0.221 & 0.049 \\
\hline $\begin{array}{l}\text { sLEPR } \\
\text { Creatinine } \\
\end{array}$ & 0.127 & 0.121 & 0.015 \\
\hline $\begin{array}{l}\text { sLEPR } \\
\text { Glucose }\end{array}$ & 0.001 & 0.265 & 0.070 \\
\hline $\begin{array}{l}\text { sLEPR } \\
\text { Cholesterol }\end{array}$ & 0.034 & 0.168 & 0.028 \\
\hline $\begin{array}{l}\text { sLEPR } \\
\text { TG } \\
\end{array}$ & 0.056 & 0.151 & 0.023 \\
\hline $\begin{array}{l}\text { sLEPR } \\
\text { ALT }\end{array}$ & 0.007 & -0.213 & 0.045 \\
\hline
\end{tabular}

Pearson's $r=$ Pearson's Regression, $\mathrm{R}^{2}=$ Coefficients of Determination

myocardial infarction (MI, heart attack) has the highest rate of morbidity and mortality. NSTEMI (non-ST elevation myocardial infarction) is a type of MI that causes partial occlusion of infarct- 

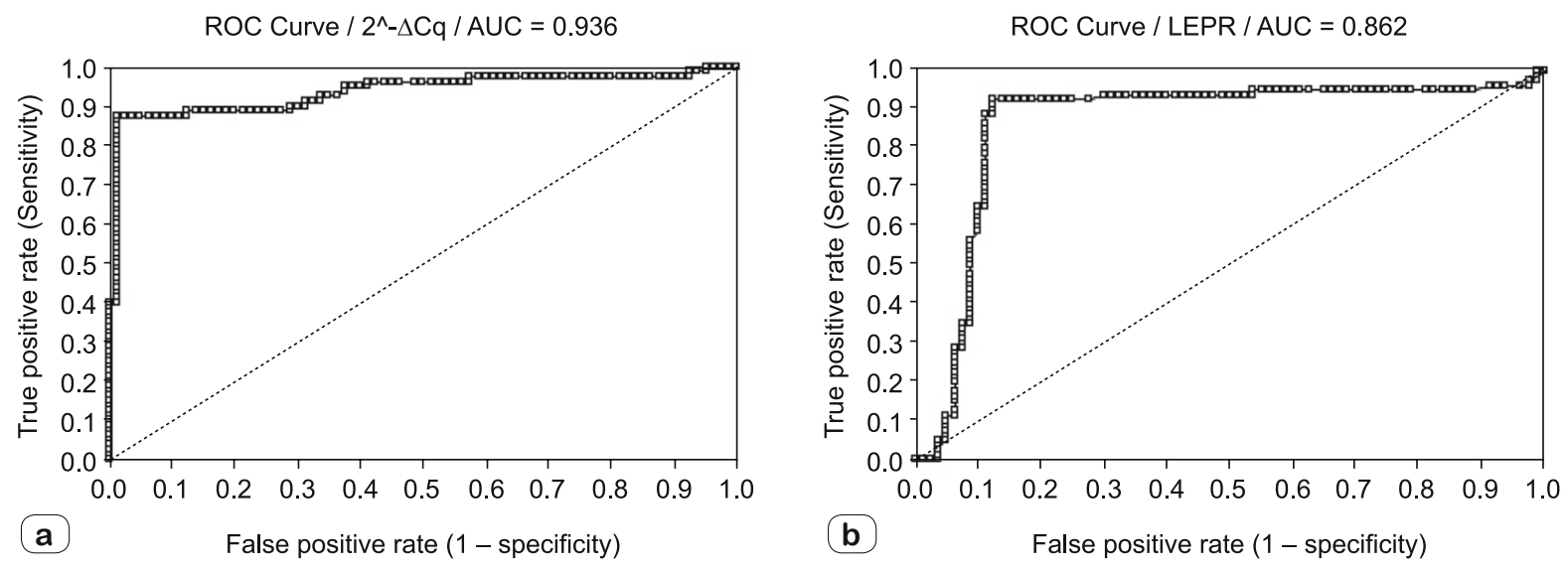

Fig. 6. The ROC curve for predicting NSTEMI. qPCR and ELISA assays was done for 80 NSTEMI patients and 80 normal controls to obtain $2^{\wedge}-\triangle C q$ of miR-4478 and sLEPR values, respectively. a) The ROC curve for serum miR-4478 (sensitivity $=87.5 \%$, specificity $=98.8 \%$, AUC $=0.936$, accuracy $=0.931,95 \%$ CI $0.395-0.476, p<0.0001$ ). b) The ROC curve for sLEPR (sensitivity $=92.5 \%$, specificity $=87.5 \%$, AUC $=$ 0.862 , accuracy $=0.900,95 \%$ CI $0.295-0.430, p<0.0001)$. AUC $=$ Area Under Curve.

ed artery. The importance of well-timed and proper recognition and management of NSTEMI is in that the partial occlusion in NSTEMI could be settled as a basement to subsequent accumulation of more substances that leads to complete occlusion of cardiac vessels and serious heart attack. Various biomarkers are used as diagnostic tools for cardiovascular diseases including NSTEMI, among them are cardiac troponins (I and T) and creatine Kinase-Muscle, Brain (CKMB). As an almost new branch of molecular biology, microRNAs brought about new strategies to quick and reliable diagnosis of various disorders, including heart diseases $(27,28)$, as their dysregulation has been found in many CVDs (29). Because of their very small sizes, microRNAs can easily cross cell membranes and appear in blood. So decrease or increase in their basal concentration in any given tissue would be reflected quickly in circulation and could be measured easily by bio molecular methods. So any pathologic status that causes changes in basal metabolism of the tissue would trigger variation in circulatory microRNA levels, much earlier than protein levels. So changes in circulatory concentration of any given microRNA could reflect metabolic alterations in the tissue that it comes from.

Human microRNA-4478 (hsa-mir-4478, miR-4478) was introduced in 2010 after sequencing of the small RNA transcriptome of normal and malignant human B cells that led to identification of hundreds of novel microRNAs (30). miR-4478 is encoded in chromosomal location of 9q33.2. Its mature form has 17 nucleotides with this sequence: 5'-gaggcugagcugaggag-3'. Soluble LEPR (sLEPR, sOB-R) resulting from cleavage of the extracellular domain of the transmembraneous leptin receptor can be present in dimeric or oligomerized states (13). sLEPR binds to leptin in ratio of $1: 1$ and modulates its biochemical action (14). Previous studies showed an increase in SLEPR levels in CVD patients (15) and miR-4478 has the highest target score $(=90)$ for LEPR (20). So we decided to test probable diminishment in miR-4478 expression as one of the reasons of increase in LEPR expression and proposee this diminishment as a new biomarker for predicting NSTEMI. So the objectives of this study were:

(a) Evaluating differences in circulatory miR-4478 levels in NSTEMI patients and healthy controls

(b) Confirming sLEPR increase in NSTEMI patients in comparison with healthy controls,

(c) Calculating the effects of changes in miR-4478 on SLEPR level, and

(d) Investigating the role of miR-4478 as a new biomarker for prediction of NSTEMI.

In the present study, we showed that: (a) Circulatory concentration of miR-4478 was significantly lower $\left(\log 2^{\wedge}-\Delta \Delta \mathrm{Cq}=-1.138 \pm\right.$ 0.569 ) but (b) sLEPR levels were statistically higher in NSTEMI patients $(30.568 \pm 3.290 \mathrm{ng} / \mathrm{dl})$ than in the control group $(23.740$ $\pm 5.457 \mathrm{ng} / \mathrm{dl})(\mathrm{F}=3.645, \mathrm{p}<0.001)$. (c) Statistically significant increases were in cTnI $(0.001), \mathrm{CKMB}(<0.001)$, Urea $(<0.001)$, Creatinine (0.009), Glucose $(<0.001)$, Cholesterol $(<0.001)$, Triglyceride (0.001), levels and decrease was in ALT level (0.018). (d) There was a correlation between $2^{\wedge}-\Delta \Delta \mathrm{Cq}$ (Fold Change in Gene Expression) and sLEPR level $\left(\mathrm{p}<0.001, \mathrm{r}=-0.835, \mathrm{R}^{2}=\right.$ 0.698 , sensitivity $=87.5 \%$, specificity $=98.8 \%$ ). (e) Both $2^{\wedge}$ $\Delta \Delta \mathrm{Cq}$ (fold change in gene expression of microRNA-4478) and sLEPR levels had high sensitivity and specificity for prediction of NSTEMI disease (sensitivity $=87.5 \%$ and specificity $=98.8 \%$ with $\mathrm{ROC}$ curve $\mathrm{AUC}=0.936$ for fold change in gene expression, and sensitivity $=92.5 \%$ and specificity $=87.5 \%$ with ROC curve $\mathrm{AUC}=0.862$ for sLEPR level). (f) There was a significant reverse correlation between serum miR-4478 concentration and circulatory levels of CKMB, and glucose. (g) There was statistically positive correlation between sLEPR and cTnI, CKMB, urea, glucose and cholesterol, and reverse correlation with ALT.

The results and statistical interpretations of our study showed for the first time that there is a decrease in circulatory miR-4478 level that is in correlation with probable subsequent increase in sLEPR concentration. Circulatory miR-4478 and leptin receptor 
$684-690$

levels are in correlation with some biofactors that are changed in NSTEMI disease, including cardiac troponin I, CKMB, cholesterol, and ALT. As LEPR and these biofactors are all important for the pathophysiology of NSTEMI as a cardiac disease, so increase in sLEPR and decrease in circulatory miR-4478 concentrations could be proposed a new biomarker for NSTEMI disease. Further studies may confirm our results by measuring intra-hepatocellular changes in miR-4478 and LEPR expression and proving these correlations.

\section{References}

1. Mortality GBD, Causes of Death C. Global, regional, and national age-sex specific all-cause and cause-specific mortality for 240 causes of death, 1990-2013: a systematic analysis for the Global Burden of Disease Study 2013. Lancet 2015; 385 (9963): 117-171.

2. Mendis S, Puska P, Norrving B. Global atlas on cardiovascular disease prevention and control. Geneva: World Health Organization, 2011.

3. Talaei M, Sarrafzadegan N, Sadeghi M, Oveisgharan S, Marshall T, Thomas GN et al. Incidence of cardiovascular diseases in an Iranian population: the Isfahan Cohort Study. Arch Iran Med 2013; 16 (3): 138-144.

4. Roger VL, Go AS, Lloyd-Jones DM, Benjamin EJ, Berry JD, Borden WB et al. Executive summary: heart disease and stroke statistics 2012 update: a report from the American Heart Association. Circulation 2012; 125 (1): 188-197.

5. Mehta PK, Wei J, Wenger NK. Ischemic heart disease in women: a focus on risk factors. Trends Cardiovasc Med 2015; 25 (2): 140-151.

6. Castelli WP. Cholesterol and lipids in the risk of coronary artery disease--the Framingham Heart Study. Can J Cardiol 1988; 4 Suppl A: $5 \mathrm{~A}-10 \mathrm{~A}$.

7. Adams JE, 3rd, Schechtman KB, Landt Y, Ladenson JH, Jaffe AS. Comparable detection of acute myocardial infarction by creatine kinase $\mathrm{MB}$ isoenzyme and cardiac troponin I. Clin Chem 1994; 40 (7 Pt 1): 1291-1295.

8. Moe KT, Wong P. Current trends in diagnostic biomarkers of acute coronary syndrome. Ann Acad Med Singapore 2010; 39 (3): 210-215.

9. Deckers JW. Classification of myocardial infarction and unstable angina: a re-assessment. Int J Cardiol 2013; 167 (6): 2387-2390.

10. Jaffe AS, Ravkilde J, Roberts R, Naslund U, Apple FS, Galvani M et al. It's time for a change to a troponin standard. Circulation 2000; 102 (11): 1216-1220.

11. Tartaglia LA, Dembski M, Weng X, Deng N, Culpepper J, Devos $\mathbf{R}$ et al. Identification and expression cloning of a leptin receptor, OB-R. Cell 1995; 83 (7): 1263-1271.

12. Tartaglia LA. The leptin receptor. J Biol Chem 1997; 272 (10): 6093-6096.

13. Lammert A, Kiess W, Bottner A, Glasow A, Kratzsch J. Soluble leptin receptor represents the main leptin binding activity in human blood. Biochem Biophys Res Commun 2001; 283 (4): 982-988.
14. Devos R, Guisez Y, Van der Heyden J, White DW, Kalai M, Fountoulakis $\mathbf{M}$ et al. Ligand-independent dimerization of the extracellular domain of the leptin receptor and determination of the stoichiometry of leptin binding. J Biol Chem 1997; 272 (29): 18304-118305.

15. Schulze PC, Kratzsch J, Linke A, Schoene N, Adams V, Gielen S et al. Elevated serum levels of leptin and soluble leptin receptor in patients with advanced chronic heart failure. Eur J Heart Fail 2003; 5 (1): 33-40.

16. Bartel DP. MicroRNAs: genomics, biogenesis, mechanism, and function. Cell 2004; 116 (2): 281-297.

17. Valencia-Sanchez MA, Liu J, Hannon GJ, Parker R. Control of translation and mRNA degradation by miRNAs and siRNAs. Genes Dev 2006; 20 (5): 515-524.

18. Hwang HW, Mendell JT. MicroRNAs in cell proliferation, cell death, and tumorigenesis. Br J Cancer 2006; 94 (6): 776-780.

19. McCarthy JJ. The MyomiR network in skeletal muscle plasticity. Exerc Sport Sci Rev 2011; 39 (3): 150-154.

20. Wang $X$. miRDB: a microRNA target prediction and functional annotation database with a wiki interface. RNA 2008; 14 (6): 1012-1017.

21. Bustin SA, Benes V, Garson JA, Hellemans J, Huggett J, Kubista $\mathbf{M}$ et al. The MIQE guidelines: minimum information for publication of quantitative real-time PCR experiments. Clin Chem 2009; 55 (4): 611-622.

22. Livak KJ, Schmittgen TD. Analysis of relative gene expression data using real-time quantitative PCR and the 2 (-Delta Delta C (T)) Method. Methods 2001; 25 (4): 402-408.

23. van Iterson M, t Hoen PA, Pedotti P, Hooiveld GJ, den Dunnen JT, van Ommen GJ et al. Relative power and sample size analysis on gene expression profiling data. BMC Genomics 2009; 10: 439.

24. Vaes E, Khan M, Mombaerts P. Statistical analysis of differential gene expression relative to a fold change threshold on NanoString data of mouse odorant receptor genes. BMC Bioinformatics 2014; 15: 39.

25. Ramakers C, Ruijter JM, Deprez RH, Moorman AF. Assumptionfree analysis of quantitative real-time polymerase chain reaction (PCR) data. Neurosci Lett 2003; 339 (1): 62-66.

26. Academic Medical Center (AMC) in Amsterdam tN. http: //www. hartfaalcentrum.nl/index.php?main=files\&sub=LinRegPCR .

27. Santovito D, Mezzetti A, Cipollone F. MicroRNAs and atherosclerosis: new actors for an old movie. Nutr Metab Cardiovasc Dis 2012; 22 (11): 937-943.

28. Small EM, Frost RJ, Olson EN. MicroRNAs add a new dimension to cardiovascular disease. Circulation 2010; 121 (8): 1022-1032.

29. Elzenaar I, Pinto YM, van Oort RJ. MicroRNAs in heart failure: new targets in disease management. Clin Pharmacol Ther 2013; 94 (4): 480-489.

30. Jima DD, Zhang J, Jacobs C, Richards KL, Dunphy CH, Choi WW et al. Deep sequencing of the small RNA transcriptome of normal and malignant human B cells identifies hundreds of novel microRNAs. Blood 2010; 116 (23): e118-127. 